\title{
Normal bases of rings of continuous functions constructed with the $\left(q_{n}\right)$-digit principle
}

by

\section{S. Evrard (Amiens)}

When $K$ is a local field with valuation ring $V$, K. Conrad [6] constructs normal bases of the $\operatorname{ring} \mathcal{C}(V, K)$ of continuous functions from $V$ to $K$, using what he calls extension by $q$-digit expansion, where $q$ denotes the cardinality of the residue field $k$ of $V$. In this article, we extend Conrad's method to the ring $\mathcal{C}(S, K)$ of continuous functions from $S$ to $K$ where $S$ denotes a subset of $V$. Moreover, we no more assume the finiteness of the residue field $k$, but replace this condition by the precompactness of $S$.

We first recall in Section 1 the notion of normal basis and Conrad's $q$-digit principle. In Section 2, we define extension by $\left(q_{n}\right)$-digit expansion. Then, in Section 3, we generalize Conrad's $q$-digit principle to a $\left(q_{n}\right)$-digit principle (Theorem 3.6), which may be applied in particular to Amice's regular compact subsets [1]. In Section 4, we end with several examples.

1. The $q$-digit principle. Let $(K,|\cdot|)$ be a complete valued nonarchimedean field. Denote by $V$ the corresponding valuation ring, $\mathfrak{M}$ its maximal ideal and $k$ its residue field. Let $(E,\|\cdot\|)$ be an ultrametric Banach space over $K$.

Definition 1.1. A sequence $\left(e_{n}\right)_{n \geq 0}$ of elements of $E$ is called a normal basis of $E$ (orthonormal basis in [6]) if

(1) each $x \in E$ has a representation as $x=\sum_{n \geq 0} x_{n} e_{n}$ where $x_{n} \in K$ and $\lim _{n \rightarrow \infty} x_{n}=0$,

(2) in the representation $x=\sum_{n \geq 0} x_{n} e_{n}$, we have $\|x\|=\sup _{n}\left|x_{n}\right|$.

2000 Mathematics Subject Classification: Primary 12J25, 11S99; Secondary 13F20, $11 \mathrm{~B} 65$.

Key words and phrases: non-archimedean valued field, regular compact, generalized Vandermonde determinant, normal bases, Legendre set.

The author wishes to thank Professor Chabert for his remarks and great help. 
Let $E_{0}=\{x \in E:\|x\| \leq 1\}$. Then $E_{0} / \mathfrak{M} E_{0}$ is a $k$-vector space. For $e_{n} \in E_{0}, \bar{e}_{n}$ denotes the reduction of $e_{n}$ modulo $\mathfrak{M} E_{0}$. The following proposition allows one to characterize normal bases in purely algebraic terms.

Proposition 1.2 ([2, Prop. 3.1.5]). Assume that the valuation is discrete and that $\|E\|=|K|$. A sequence $\left(e_{n}\right)_{n \in \mathbb{N}}$ of elements of $E$ is a normal basis of $E$ if and only if $e_{n} \in E_{0}$ for every $n \geq 0$ and $\left(\bar{e}_{n}\right)_{n \in \mathbb{N}}$ is a k-basis of $E_{0} / \mathfrak{M} E_{0}$.

Assuming that $k$ is finite with cardinality $q$ (hence $K$ is a local field), $\mathrm{K}$. Conrad [6] uses extension by $q$-digit expansion to construct some normal bases of the ring $\mathcal{C}(V, K)$. We first recall this notion.

Definition 1.3. Let $\left(e_{n}\right)_{n \geq 0}$ be a sequence of elements of $\mathcal{C}(V, V)$. We construct another sequence of functions $\left(f_{i}\right)$ in the following way:

$$
\text { if } i=i_{0}+i_{1} q+\cdots+i_{r} q^{r}\left(0 \leq i_{j}<q\right) \text { then } f_{i}=e_{0}^{i_{0}} \cdots e_{r}^{i_{r}} .
$$

The sequence $\left(f_{i}\right)$ is called the extension of $\left(e_{n}\right)$ by q-digit expansion.

In characteristic $p, V$ contains a field which is isomorphic to $k$, and so it may be viewed as a $k$-vector space. In this case, the $q$-digit principle has the following form:

Proposition 1.4 (Digit principle in characteristic $p$ [6, Theorem 2]). If the sequence $\left(e_{n}\right)$ is a normal basis of the ring of continuous $k$-linear functions from $V$ to $K$, then the extension of $\left(e_{n}\right)$ by q-digit expansion is a normal basis of $\mathcal{C}(V, K)$.

As noted by K. Conrad, in characteristic 0 there is no analogue of the subspace of linear functions. Nevertheless, there is another version that holds in any characteristic:

Proposition 1.5 (Digit principle in any characteristic [6, Theorem 3]). Let $\left(e_{n}\right)_{n \geq 0}$ be a sequence of elements of $\mathcal{C}(V, V)$ such that the reductions $\bar{e}_{i} \in \mathcal{C}(V, \bar{k})$ are constant on cosets modulo $\mathfrak{M}^{i+1}$ and the map

$$
\phi_{n}: V / \mathfrak{M}^{n} \rightarrow k^{n}, \quad x \mapsto\left(\bar{e}_{0}(x), \ldots, \bar{e}_{n-1}(x)\right),
$$

is bijective. Then the extension of $\left(e_{n}\right)$ by q-digit expansion is a normal basis of $\mathcal{C}(V, K)$.

To generalize the $q$-digit principle to subsets $S$, the map $\phi_{r}$ will be required to be only injective, as $S / \mathfrak{M}^{r}$ does not necessarily contain $q^{r}$ elements.

2. The $\left(q_{n}\right)$-digit expansion. Hypotheses and notation. Let $V$ be a discrete valuation domain, with valuation $v$. Denote by $K$ the quotient field of $V$, by $\mathfrak{M}$ the maximal ideal of $V$, by $\pi$ a generator of $\mathfrak{M}$ (with $v(\pi)=1$ ), by $k=V / \mathfrak{M}$ the residue field and by $q$ the cardinality (finite or not) of $k$. Let $S$ be an infinite subset of $V$. 
We denote by $\widehat{V}, \widehat{K}$, and $\widehat{S}$ the completions of $V, K$ and $S$ with respect to the $\mathfrak{M}$-adic topology. We still denote by $v$ the extension of $v$ to $\widehat{K}$. For every $n \geq 0$, we denote by $S / \mathfrak{M}^{n}$ the set formed by the classes of $S$ modulo $\mathfrak{M}^{n}$ and we define $q_{n}$ to be the cardinality of $S / \mathfrak{M}^{n}\left(q_{0}=1\right)$.

We assume that $S$ is precompact, that is, $\widehat{S}$ is compact, and we know that this is equivalent to the fact that all the $q_{n}$ 's are finite.

Of course, $\left(q_{n}\right)$ is a non-decreasing and non-stationary sequence. Now, we define the $\left(q_{n}\right)$-digit expansion of a positive integer $m$ :

Proposition 2.1. Let $\left(q_{n}\right)_{n \geq 0}$ be a non-decreasing and non-stationary sequence of integers, with $q_{0}=1$. For every $m>0$, there exists a unique representation of $m$ as

$$
m=m_{0}+m_{1} q_{1}+\cdots+m_{r} q_{r}
$$

where $r$ is such that

$$
q_{r} \leq m<q_{r+1}
$$

and where, for every $j$ in $[1, r]$,

$$
m_{j} \geq 0 \quad \text { and } m_{0}+m_{1} q_{1}+\cdots+m_{j} q_{j}<q_{j+1} .
$$

This representation is called the $\left(q_{n}\right)$-digit expansion of $m$.

Proof. Suppose there is such a representation of $m$. For $0 \leq k \leq r$, let

$$
N_{k}=m_{0}+m_{1} q_{1}+\cdots+m_{k} q_{k} .
$$

Hence, for $1 \leq k \leq r$, one has

$$
N_{k}=N_{k-1}+m_{k} q_{k} \quad \text { with } \quad N_{k-1}<q_{k} .
$$

So, $m_{k}$ is the quotient of the division of $N_{k}$ by $q_{k}$, and $N_{k-1}$ is the rest. Consequently, the sequence $\left(m_{k}\right)$ is uniquely determined.

Conversely, let us prove that such a sequence satisfies our hypothesis. Consider the sequences $N_{r}, N_{r-1}, \ldots, N_{0}$ and $m_{r}, m_{r-1}, \ldots, m_{0}$ defined by induction in the following way:

$$
\begin{cases}N_{r}=m, & \\ m_{k}=\left[N_{k} / q_{k}\right] & \text { for } 0 \leq k \leq r, \\ N_{k-1}=N_{k}-m_{k} q_{k} & \text { for } 1 \leq k \leq r .\end{cases}
$$

By definition of $r, m_{r}=\left[m / q_{r}\right] \neq 0$. At each step $(1 \leq k \leq r)$, one has $N_{k-1}<q_{k}$ and $m=N_{k-1}+m_{k} q_{k}+\cdots+m_{r} q_{r}$. Indeed,

$$
\sum_{l=k}^{r} m_{l} q_{l}=\sum_{l=k}^{r}\left(N_{l}-N_{l-1}\right)=m-N_{k-1} .
$$

Hence,

$$
m=N_{0}+m_{1} q_{1}+\cdots+m_{r} q_{r}, \quad m_{0}=\left[\frac{N_{0}}{q_{0}}\right]=N_{0} .
$$


Finally, $m=\sum_{k=0}^{r} m_{k} q_{k}$ and, for $0 \leq k \leq r$, $m_{0}+m_{1} q_{1}+\cdots+m_{k} q_{k}=m-\left(m_{k+1} q_{k+1}+\cdots+m_{r} q_{r}\right)=N_{k}<q_{k+1}$.

REMARKS 2.2 .

(1) Let $m=m_{0}+m_{1} q_{1}+\cdots+m_{r} q_{r}$ be the $\left(q_{n}\right)$-digit expansion of $m$. Then, for $0 \leq j \leq r$, one has:

- $0 \leq m_{j}<q_{j+1} / q_{j}$,

- in particular, if $q_{j}=q_{j+1}$ then $m_{j}=0$.

(2) The condition $0 \leq m_{j}<q_{j+1} / q_{j}$ is not sufficient to define the $m_{j}$ 's. If we consider the sequence $q_{n}=2 n+1$ of odd integers, the $\left(q_{n}\right)$ digit expansion of $m=5$ is $m=5=q_{2}$, but one can also write $m=2+3=2 q_{0}+q_{1}$ with $m_{0}=2<q_{1} / q_{0}=3$.

(3) On the contrary, the condition $0 \leq m_{j}<q_{j+1} / q_{j}$ does characterize the $\left(q_{n}\right)$-digit expansion when $q_{j}$ divides $q_{j+1}$. Indeed, if $\alpha_{j}=q_{j+1} / q_{j}$ is an integer and $0 \leq m_{j}<\alpha_{j}$, then $m_{0}<q_{1}$, and by induction, $\left(m_{0}+m_{1} q_{1}+\cdots+m_{j-1} q_{j-1}\right)+m_{j} q_{j}<q_{j}+\left(\alpha_{j}-1\right) q_{j}=\alpha_{j} q_{j}=q_{j+1}$.

(4) If the sequence $\left(q_{n}\right)$ is associated to a subset $S$ (that is, $q_{n}=$ $\left.\operatorname{card}\left(S / \mathfrak{M}^{n}\right)\right)$, then we have $q_{n} \leq q_{n+1} \leq q q_{n}$. As already said, $\left(q_{n}\right)$ is a non-decreasing and non-stationary sequence. Note that it need not be strictly increasing and $q_{n}$ does not necessarily divide $q_{n+1}$, as shown by $V=\mathbb{Z}_{5}$ and $S=125 \mathbb{Z}_{5} \cup\left\{25+125 \mathbb{Z}_{5}\right\} \cup\left\{1+125 \mathbb{Z}_{5}\right\}$. One has: $S /(5)=\{0,1\}$ and $q_{1}=2 ; S /(25)=\{0,1\}$ and $q_{2}=2$; $S /(125)=\{0,1,25\}$ and $q_{3}=3 ; q_{4}=15$ and, more generally, $q_{n}=3 \cdot 5^{n-3}$ for $n \geq 3$.

Definition 2.3. Let $\left(e_{n}\right)_{n \geq 0}$ be a sequence of elements of a commutative monoid (with an identity element). The extension of the sequence $\left(e_{n}\right)_{n \geq 0}$ by $\left(q_{n}\right)$-digit expansion is the following sequence $\left(f_{m}\right)_{m \geq 0}$ :

$$
f_{m}=e_{0}^{m_{0}} \times e_{1}^{m_{1}} \times \cdots \times e_{r}^{m_{r}}
$$

where $m=m_{0}+m_{1} q_{1}+\cdots+m_{r} q_{r}$ is the $\left(q_{n}\right)$-digit expansion of $m$.

REMARKS 2.4 .

(1) $f_{0}=1$

(2) If there exists $j$ such that $q_{j}=q_{j+1}$, then the term $e_{j}$ of the sequence $\left(e_{n}\right)$ never appears in any element of the sequence $\left(f_{m}\right)$.

(3) For $q_{r} \leq m<q_{r+1}$, if $m=m_{r} q_{r}+N_{r}$ with $N_{r}<q_{r}$, then

$$
f_{m}=e_{r}^{m_{r}} \times f_{N_{r}} .
$$

We now try to find conditions on the subset $S$ and on the sequence $\left(e_{n}\right)_{n \geq 0}$ of elements of $\mathcal{C}(\widehat{S}, \widehat{V})$ for the sequence $\left(f_{m}\right)_{m \geq 0}$ to be a normal basis of $\mathcal{C}(\widehat{S}, \widehat{K})$. We first assume that the sequence $\left(e_{n}\right)_{n \geq 0}$ satisfies a condition similar to that considered by K. Conrad. More precisely, let $\left(e_{n}\right)_{n \geq 0}$ be a 
sequence of elements of $\mathcal{C}(\widehat{S}, \widehat{V})$ such that, for each $n \geq 0$, the reduction $\bar{e}_{n}$ of $e_{n}$ in $\mathcal{C}(\widehat{S}, k)$ is constant on cosets of $S$ modulo $\mathfrak{M}^{n+1}$. Denote by $\left(f_{m}\right)_{m \geq 0}$ the extension of $\left(e_{n}\right)_{n \geq 0}$ by $\left(q_{n}\right)$-digit expansion. It is obvious that, for $0 \leq m<q_{r}$, the reductions $\bar{f}_{m}$ in $\mathcal{C}(\widehat{S}, k)$ are constant on cosets of $S$ modulo $\mathfrak{M}^{r}$. In order to determine when this sequence is a normal basis of $\mathcal{C}(\widehat{S}, \widehat{K})$, we use the following lemma.

LEMMA $2.5([8])$. Let $\left(g_{n}\right)_{n \geq 0}$ be a sequence of $\mathcal{C}(\widehat{S}, \widehat{V})$ such that, for $0 \leq$ $m<q_{r}$, the reductions $\bar{g}_{m}$ in $\mathcal{C}(\widehat{S}, k)$ are constant on cosets of $S$ modulo $\mathfrak{M}^{r}$. The following assertions are equivalent:

(1) $\left(g_{n}\right)$ is a normal basis of $\mathcal{C}(\widehat{S}, \widehat{K})$,

(2) $\left(\bar{g}_{n}\right)$ is a $k$-linear basis of $\mathcal{C}(\widehat{S}, k)$,

(3) for each integer $r \geq 1,\left(\bar{g}_{m}\right)_{0 \leq m<q_{r}}$ is a $k$-basis of $\mathcal{F}\left(S / \mathfrak{M}^{r}, k\right)$, the space of functions from $S / \mathfrak{M}^{r}$ to $k$,

(4) for each $n$, the $\bar{g}_{m}$ 's $(0 \leq m<n)$ are $k$-linearly independent.

Proof. Proposition 1.2 gives the equivalence between assertions (1) and (2). The equivalence between (3) and (4) follows from the dimension of the vector space $\mathcal{F}\left(S / \mathfrak{M}^{r}, k\right)$. Obviously, (2) implies (4). Finally, (3) implies (2), as a continuous function from $\widehat{S}$ to $k$ is locally constant and can be viewed as a map from $S / \mathfrak{M}^{r}$ to $k$ for some $r$.

Proposition 2.6. Let $\left(g_{n}\right)_{n \geq 0}$ be a sequence of functions such that, for every $0 \leq m<q_{r}$, the reductions $\bar{g}_{m}$ in $\mathcal{C}(\widehat{S}, k)$ are constant on cosets of $S$ modulo $\mathfrak{M}^{r}$. For $r \geq 1$, let $G_{r}$ be the following matrix:

$$
G_{r}=\left(\bar{g}_{j}\left(a_{i}\right)\right)_{0 \leq i, j<q_{r}},
$$

where $\left(a_{i}\right)_{0 \leq i<q_{r}}$ denotes a complete set of residues of $S$ modulo $\mathfrak{M}^{r}$. Then:

(1) $\operatorname{det} G_{r}$ does not depend on the $a_{i}$ 's (except for the sign).

(2) The $\bar{g}_{m}$ 's $\left(0 \leq m<q_{r}\right)$ are $k$-linearly independent if and only if $\operatorname{det} G_{r} \neq 0$.

Proof. (1) If $\left(b_{i}\right)_{0 \leq i<q_{r}}$ is another complete set of residues of $S$ modulo $\mathfrak{M}^{r}$, there exists a permutation $\sigma$ such that $b_{i} \equiv a_{\sigma(i)}\left(\bmod \mathfrak{M}^{r}\right)$. As the $\bar{g}_{j}$ 's are constant on cosets of $S$ modulo $\mathfrak{M}^{r}$, the sets of rows of $\left(\bar{g}_{j}\left(a_{i}\right)\right)_{0 \leq i, j<q_{r}}$ and of $\left(\bar{g}_{j}\left(b_{i}\right)\right)_{0 \leq i, j<q_{r}}$ are permutations of each other.

(2) Suppose that the $\lambda_{m} \in k\left(0 \leq m<q_{r}\right)$ are such that

$$
\lambda_{0} \bar{g}_{0}+\lambda_{1} \bar{g}_{1}+\cdots+\lambda_{q_{r}-1} \bar{g}_{q_{r}-1}=0 .
$$

Evaluating the $g_{m}$ 's $\left(0 \leq m<q_{r}\right)$ on the $q_{r}$ elements of $S / \mathfrak{M}^{r}$, we obtain a system of $q_{r}$ equations in the $q_{r}$ unknowns $\lambda_{m}$. This system has a unique solution if and only if $\operatorname{det} G_{r} \neq 0$. 
3. Normal basis obtained by the $\left(q_{n}\right)$-digit principle. We still maintain the hypotheses and notation introduced in Section 2 and we complete them by the following:

Hypotheses and notation. Let $r \in \mathbb{N}$ be fixed and denote by $\left(a_{i}\right)_{0 \leq i<q_{r+1}}$ a complete set of residues of $S$ modulo $\mathfrak{M}^{r+1}$ such that $\left(a_{i}\right)_{0 \leq i<q_{r}}$ is a complete set of residues of $S$ modulo $\mathfrak{M}^{r}$. For $0 \leq i<q_{r}$, let

$$
\gamma_{i}=\operatorname{card}\left\{j: 0 \leq j<q_{r+1}, a_{j} \equiv a_{i}\left(\bmod \mathfrak{M}^{r}\right)\right\} .
$$

Moreover, we order the $a_{i}$ 's $\left(0 \leq i<q_{r}\right)$ so that

$$
\gamma_{0} \geq \cdots \geq \gamma_{q_{r}-1} \geq 1 \text {. }
$$

Let $\left(e_{n}\right)_{n \geq 0}$ be a sequence of elements of $\mathcal{C}(\widehat{S}, \widehat{V})$ such that, for each $n \geq 0$, the reduction $\bar{e}_{n}$ of $e_{n}$ in $\mathcal{C}(\widehat{S}, k)$ is constant on cosets of $S$ modulo $\mathfrak{M}^{n+1}$. Denote by $\left(f_{m}\right)_{m \geq 0}$ the extension of $\left(e_{n}\right)_{n \geq 0}$ by $\left(q_{n}\right)$-digit expansion. Clearly, we have:

Lemma 3.1. There are exactly $\gamma_{q_{r}-1}$ complete sets of residues of $S$ modulo $\mathfrak{M}^{r}$ in a complete set of residues of $S / \mathfrak{M}^{r+1}$. Moreover, for all $0 \leq i, j$ $<q_{r+1}$ such that $a_{i} \equiv a_{j}\left(\bmod \mathfrak{M}^{r}\right)$, one has:

(1) $\forall k<r, \bar{e}_{k}\left(a_{i}\right)=\bar{e}_{k}\left(a_{j}\right)$,

(2) $\forall k<q_{r}, \bar{f}_{k}\left(a_{i}\right)=\bar{f}_{k}\left(a_{j}\right)$.

\subsection{A necessary condition}

Lemma 3.2. Suppose that there exists $r$ such that $q_{r}$ divides $q_{r+1}$ and write $q_{r+1}=\alpha_{r} q_{r}$. If the $\bar{f}_{m}$ 's $\left(0 \leq m<q_{r+1}\right)$ are $k$-linearly independent, then

$$
\gamma_{0}=\gamma_{1}=\cdots=\gamma_{q_{r}-1}=\alpha_{r}=q_{r+1} / q_{r} .
$$

Proof. Assume that $\gamma_{0}>\alpha_{r}$. First, note that $q_{r}<q_{r+1}$ since, if $q_{r}=q_{r+1}$, one has $\gamma_{i}=1=\alpha_{r}$ for every $i$. In the matrix $G_{r+1}=\left(\bar{f}_{j}\left(a_{i}\right)\right)_{0 \leq i, j<q_{r+1}}$, we arrange the columns into the following sequence:

$$
1, \bar{e}_{r}, \ldots, \bar{e}_{r}^{\alpha_{r}-1}, \bar{f}_{1}, \ldots, \bar{f}_{1} \bar{e}_{r}^{\alpha_{r}-1}, \ldots, \bar{f}_{i} \bar{e}_{r}^{j}, \ldots, \bar{f}_{q_{r}-1} \bar{e}_{r}^{\alpha_{r}-1} .
$$

We denote by $C_{i, j}$ the column corresponding to $\bar{f}_{i} \bar{e}_{r}^{j}$ and, for $1 \leq i<q_{r}$ and $0 \leq j<\alpha_{r}$, we use the following elementary transformations on columns:

$$
C_{i, j} \leftarrow C_{i, j}-\bar{f}_{i}\left(a_{0}\right) C_{0, j} .
$$

For $1 \leq l<q_{r+1}$, the term in the column $C_{i, j}$ and the row $L_{l}$ becomes

$$
\bar{f}_{i}\left(a_{l}\right) \bar{e}_{r}^{j}\left(a_{l}\right)-\bar{f}_{i}\left(a_{0}\right) \bar{e}_{r}^{j}\left(a_{l}\right) .
$$

It follows from Lemma 3.1 that, whenever $l\left(0 \leq l<q_{r+1}\right)$ is such that $a_{l} \equiv a_{0}\left(\bmod \mathfrak{M}^{r}\right)$, then $\bar{f}_{i}\left(a_{0}\right)=\bar{f}_{i}\left(a_{l}\right)$ and, after permuting the rows of the matrix, the first $\gamma_{0}$ new rows (corresponding to such an $a_{l}$ ) end with 
zeros. Consequently, the new matrix is of the form

$$
\left(\begin{array}{c|c}
A & 0 \\
B & C
\end{array}\right) \quad \text { where } \quad A \in M_{\alpha_{r}}(k),
$$

and, as $\gamma_{0}>\alpha_{r}$, the first line of $C$ is null. Finally,

$$
\operatorname{det} G_{r+1}=\operatorname{det} A \cdot \operatorname{det} C=0 .
$$

This necessary condition defines a class of subsets of $V$ called Legendre subsets in [7]. Before stating our main theorem, we recall some properties of these sets.

3.2. Legendre sets

Definition 3.3. The subset $S$ is called a Legendre set if, for every $r$ in $\mathbb{N}$, each class of $S$ modulo $\mathfrak{M}^{r}$ contains the same number of elements modulo $\mathfrak{M}^{r+1}$.

If $S$ is a Legendre set then, for every $r \geq 0, q_{r}$ divides $q_{r+1}$ and for every $0 \leq i<q_{r}$, one has

$$
\gamma_{i}=q_{r+1} / q_{r}
$$

Such subsets have been studied by Y. Amice [1] as regular compact subsets in the case when $K$ is a local field and $S$ is compact, and by Y. Fares and the author [7] in a more general setting. Let us recall a property of the Legendre sets that we will use in the applications. We first recall the following definitions:

Definition 3.4. Let $\left(a_{n}\right)_{n \geq 0}$ be a sequence of elements of $S$.

(1) The sequence is called a $v$-ordering of $S$ (see [3]) when, for every $n>0$,

$$
v\left(\prod_{0 \leq k<n}\left(a_{n}-a_{k}\right)\right)=\inf _{x \in S} v\left(\prod_{0 \leq k<n}\left(x-a_{k}\right)\right) .
$$

(2) The sequence is called a very well distributed sequence of $S$ (see [1]) if, for every $r>0$ and every $\lambda \in \mathbb{N},\left(a_{\lambda q_{r}}, \ldots, a_{(\lambda+1) q_{r}-1}\right)$ is a complete set of residues of $S / \mathfrak{M}^{r}$.

We then have a very nice property:

Proposition 3.5 ([7]).

- A very well distributed sequence of a subset is a v-ordering.

- Every v-ordering of a Legendre set is a very well distributed sequence.

Here are some examples of Legendre sets:

Example 1. Assume that the residue field $k$ is finite of cardinality $q$.

(1) $V$ is a Legendre set and $q_{n}=q q_{n-1}=q^{n}$. 
(2) Let $S=\bigcup_{j=1}^{r} b_{j}+\mathfrak{M}$, where $b_{1}, \ldots, b_{r}$ are not congruent modulo $\mathfrak{M}$. Then $S$ is a Legendre set and $q_{n}=r q^{n-1}$.

(3) Let $u \in V$ be such that $v(u)=0$. Then $S=\left\{u^{n}: n \in \mathbb{N}\right\}$ is a Legendre set.

We are ready to state our theorem.

3.3. Extension of Conrad's q-digit principle

TheOREM 3.6. Let $V$ be a discrete valuation domain with maximal ideal $\mathfrak{M}$ and residue field $k=V / \mathfrak{M}$. Let $S$ be a precompact subset of $V$ and, for $n \geq 0$, let $q_{n}=\operatorname{card}\left(S / \mathfrak{M}^{n}\right)$. Assume that, for every $r, q_{r}$ divides $q_{r+1}$. Let $\left(e_{i}\right)$ be a sequence of elements of $\mathcal{C}(\widehat{S}, \widehat{V})$ such that the reductions $\bar{e}_{i} \in \mathcal{C}(\widehat{S}, k)$ are constant on cosets of $S$ modulo $\mathfrak{M}^{i+1}$ and suppose that, for every $r \geq 0$, the following map is injective:

$$
\phi_{r}: S / \mathfrak{M}^{r+1} \rightarrow k^{r+1}, \quad x \mapsto\left(\bar{e}_{0}(x), \ldots, \bar{e}_{r}(x)\right) .
$$

Then the extension $\left(f_{m}\right)_{m \geq 0}$ of $\left(e_{n}\right)_{n \geq 0}$ by $\left(q_{n}\right)$-digit expansion is a normal basis of $\mathcal{C}(\widehat{S}, \widehat{K})$ if and only if $S$ is a Legendre set.

Proof. The necessity follows from Lemmas 2.5 and 3.2. Using Proposition 2.6, we now show that the condition is sufficient. We prove by induction on $r$ that $\operatorname{det} G_{r} \neq 0$. For $r=0$, one has

$$
\operatorname{det} G_{1}=V\left(\bar{e}_{0}\left(a_{0}\right), \ldots, \bar{e}_{0}\left(a_{q_{1}-1}\right)\right)
$$

where $V(\cdot)$ denotes the Vandermonde determinant. By hypothesis, $\phi_{0}$ is injective, hence $\operatorname{det} G_{1} \neq 0$. Now, we suppose that $\operatorname{det} G_{r} \neq 0$ and we show that $\operatorname{det} G_{r+1} \neq 0$. First, as there are exactly $\alpha_{r}$ complete sets of residues of $S$ modulo $\mathfrak{M}^{r}$ in $\left(a_{i}\right)_{0 \leq i<q_{r}}$, we can assume that for $0 \leq i<q_{r}$ and $0 \leq l<\alpha_{r}$,

$$
a_{i+l q_{r}} \equiv a_{i}\left(\bmod \mathfrak{M}^{r}\right) .
$$

Then we compute $\operatorname{det} G_{r+1}$ by ordering each row $L_{r+1}$ in the matrix as follows:

$$
L_{1}=\left(\bar{f}_{0}, \ldots, \bar{f}_{q_{1}-1}\right)=\left(1, \bar{e}_{0}, \ldots, \bar{e}_{0}^{q_{1}-1}\right)
$$

and, for $r \geq 1$,

$$
L_{r+1}=\left(L_{r}, \bar{e}_{r} L_{r}, \ldots, \bar{e}_{r}^{\alpha_{r}-1} L_{r}\right) .
$$

So we can write

$$
G_{r+1}=\left(\begin{array}{cccc}
I_{q_{r}} & J_{0} & \ldots & J_{0}^{\alpha_{r}-1} \\
\vdots & J_{1} & \ldots & J_{1}^{\alpha_{r}-1} \\
\vdots & \vdots & \vdots & \vdots \\
I_{q_{r}} & J_{\alpha_{r}-1} & \ldots & J_{\alpha_{r}-1}^{\alpha_{r}-1}
\end{array}\right) \cdot\left(\begin{array}{ccc}
G_{r} & 0 & 0 \\
0 & \ddots & 0 \\
0 & 0 & G_{r}
\end{array}\right)
$$


with, for $0 \leq l<\alpha_{r}$,

$$
J_{l}=\left(\begin{array}{ccc}
\bar{e}_{r}\left(a_{l q_{r}}\right) & 0 & 0 \\
0 & \ddots & 0 \\
0 & 0 & \bar{e}_{r}\left(a_{(l+1) q_{r}-1}\right)
\end{array}\right)
$$

We now compute the determinant of $B$, noticing that the matrices $J_{l}$ and $J_{j}$ commute:

$$
\operatorname{det} B=V\left(J_{0}, \ldots, J_{\alpha_{r}-1}\right)=\prod_{0 \leq l<j<\alpha_{r}} \operatorname{det}\left(J_{j}-J_{l}\right) .
$$

We then obtain

$$
\operatorname{det} G_{r+1}=\operatorname{det} G_{r}^{\alpha_{r}} \cdot \prod_{i=0}^{q_{r}-1} V\left(\bar{e}_{r}\left(a_{i}\right), \bar{e}_{r}\left(a_{q_{r}+i}\right), \ldots, \bar{e}_{r}\left(a_{\left(\alpha_{r}-1\right) q_{r}+i}\right)\right) .
$$

By induction hypothesis, $\operatorname{det} G_{r} \neq 0$. Moreover, as

$$
\bar{e}_{j}\left(a_{i}\right)=\bar{e}_{j}\left(a_{l q_{r}+i}\right) \quad \text { for } j<r \text { and } 0 \leq l<\alpha_{r},
$$

the injectivity of $\phi_{r+1}$ implies that

$$
\bar{e}_{r}\left(a_{i+j q_{r}}\right) \neq \bar{e}_{r}\left(a_{i+l q_{r}}\right) \quad \text { for } 0 \leq j<l \leq \alpha_{r} .
$$

Hence,

$$
V\left(\bar{e}_{r}\left(a_{i}\right), \bar{e}_{r}\left(a_{q_{r}+i}\right), \ldots, \bar{e}_{r}\left(a_{\left(\alpha_{r}-1\right) q_{r}+i}\right)\right) \neq 0 \quad \text { for } 1 \leq i \leq q_{r}
$$

\section{Applications}

4.1. Examples of normal bases obtained by the $\left(q_{n}\right)$-digit principle. For the following examples, the hypotheses of Theorem 3.6 are clearly satisfied.

Proposition 4.1. Let $S$ be a Legendre set, and denote by $F$ a complete set of residues of $V$ modulo $\mathfrak{M}$. Each $x$ in $S$ has a unique representation of the form $x=x_{0}+x_{1} \pi+\cdots+x_{j} \pi^{j}+\cdots$ with $x_{j} \in F$. For each $j \geq 0$, let

$$
\omega_{j}: S \rightarrow V, \quad x \mapsto x_{j}
$$

Then $\left(\Omega_{m}\right)$, the extension of $\left(\omega_{n}\right)$ by $\left(q_{n}\right)$-digit expansion, is a normal basis of $\mathcal{C}(\widehat{S}, \widehat{K})$.

The second example uses hyperdifferential operators as defined by Voloch in [9]: We suppose here that the characteristic of $V$ is $p>0$, so we can consider $V$ as a $k$-vector space. He defines a sequence of $k$-linear maps $\delta_{r}$ by the following condition:

$$
\forall r \in \mathbb{N}, \forall m \in \mathbb{N}, \quad \delta_{r}\left(\pi^{m}\right)=\left(\begin{array}{c}
m \\
r
\end{array}\right) \pi^{m-r} .
$$


Proposition 4.2. Let $S$ be a Legendre set of $V$. Then the extension $\left(\Delta_{m}\right)$ of $\left(\delta_{r}\right)$ by $\left(q_{n}\right)$-digit expansion is a normal basis of $\mathcal{C}(\widehat{S}, \widehat{K})$.

4.2. A polynomial example. We end with a polynomial example. We already know ([5] or [4]) that, if $S$ is a subset in a discrete valuation ring $V$ and $\left(a_{n}\right)_{n \geq 0}$ is a $v$-ordering of $S$, then the sequence of polynomials

$$
u_{r}(X)=\prod_{0 \leq i<r} \frac{X-a_{i}}{a_{r}-a_{i}}
$$

is a normal basis of $\mathcal{C}(\widehat{S}, \widehat{K})$. Here is another example:

Proposition 4.3. Let $S$ be a Legendre set and $\left(a_{n}\right)_{n \geq 0}$ be a v-ordering of $S$. Let $\left(e_{r}\right)$ be defined by

$$
e_{0}(X)=X, \quad e_{r}(X)=\prod_{0 \leq i<q_{r}} \frac{X-a_{i}}{a_{q_{r}}-a_{i}} \quad \text { for } r \geq 1 .
$$

Then the extension $\left(f_{m}\right)$ of $\left(e_{r}\right)$ by $\left(q_{n}\right)$-digit expansion is a normal basis of $\mathcal{C}(\widehat{S}, \widehat{K})$.

Proof. Of course, $e_{r}$ is an integer-valued polynomial with $\operatorname{deg}\left(e_{r}\right)=q_{r}$. First, we prove that for every $r, \bar{e}_{r} \in \mathcal{C}(\widehat{S}, k)$ is constant on cosets of $S$ modulo $\mathfrak{M}^{r+1}$. As recalled in Proposition 3.5, every $v$-ordering of a Legendre set $S$ is very well distributed in $S$. So, for each $x$ in $S$, there exists a unique $s$ such that $0 \leq s<q_{r+1}$ and $x \equiv a_{s}\left(\bmod \mathfrak{M}^{r+1}\right)$. We have to prove that

$$
\bar{e}_{r}(x)=\bar{e}_{r}\left(a_{s}\right) .
$$

First suppose that $s \geq q_{r}$. Then

$$
\forall i \in\left\{0, \ldots, q_{r}-1\right\}, \quad \frac{x-a_{i}}{a_{s}-a_{i}}=1+\frac{x-a_{s}}{a_{s}-a_{i}} .
$$

As $v\left(x-a_{s}\right) \geq r+1$ and $v\left(a_{s}-a_{i}\right)<r+1$, we have

$$
\frac{x-a_{s}}{a_{s}-a_{i}} \equiv 0(\bmod \mathfrak{M}) \quad \text { and } \quad \prod_{0 \leq i \leq q_{r}-1} \frac{x-a_{i}}{a_{s}-a_{i}} \equiv 1(\bmod \mathfrak{M}) .
$$

To conclude, write

$$
e_{r}(x)=e_{r}\left(a_{s}\right) \cdot \prod_{0 \leq i<q_{r}} \frac{x-a_{i}}{a_{s}-a_{i}} .
$$

Then $e_{r}(x) \equiv e_{r}\left(a_{s}\right)(\bmod \mathfrak{M})$.

Suppose now that $s<q_{r}$. Then $\bar{e}_{r}\left(a_{s}\right)=0$. If we had

$$
v\left(\prod_{0 \leq i<q_{r}}\left(x-a_{i}\right)\right)=v\left(\prod_{0 \leq i<q_{r}}\left(a_{q_{r}}-a_{i}\right)\right),
$$


then $x$ could replace $a_{q_{r}}$ in a $v$-ordering. Meanwhile, we could construct a new $v$-ordering

$$
a_{0}, \ldots, a_{q_{r}-1}, x, b_{q_{r}+1}, \ldots, b_{q_{r+1}-1}, \ldots
$$

Since a $v$-ordering must be a very well distributed sequence,

$$
a_{0}, \ldots, a_{q_{r}-1}, x, b_{q_{r}+1}, \ldots, b_{q_{r+1}-1}
$$

must be a complete set of residues modulo $\mathfrak{M}^{r+1}$. This is impossible, since $v\left(x-a_{s}\right) \geq r+1$. So

$$
v\left(\prod_{0 \leq i<q_{r}}\left(x-a_{i}\right)\right)>v\left(\prod_{0 \leq i<q_{r}}\left(a_{q_{r}}-a_{i}\right)\right) \text { and } \bar{e}_{r}(x)=0 .
$$

We now prove by induction on $r$ that the $\phi_{r}$ 's are injective. This is equivalent to proving that

$$
\Phi_{r}(x)=\Phi_{r}(y) \Rightarrow x \equiv y\left(\bmod \mathfrak{M}^{r+1}\right),
$$

where

$$
\Phi_{r}: S \rightarrow k^{r+1}, \quad x \mapsto\left(\bar{e}_{0}(x), \ldots, \bar{e}_{r}(x)\right) .
$$

Since $\bar{e}_{0}(X)=X$, clearly $\bar{e}_{0}(x)=\bar{e}_{0}(y)$ implies $x \equiv y(\bmod \mathfrak{M})$, so $\phi_{0}$ is injective. Now suppose that $\phi_{r-1}$ is injective. If $x \not \equiv y\left(\bmod \mathfrak{M}^{r}\right)$, it follows by induction that $\Phi_{r-1}(x) \neq \Phi_{r-1}(y)$ and then $\Phi_{r}(x) \neq \Phi_{r}(y)$. Thus we may assume that $x$ and $y$ are both in the class of some $a_{j}\left(j<q_{r}\right)$ modulo $\mathfrak{M}^{r}$ :

$$
x=a_{j}+b \pi^{r} \text { and } y=a_{j}+c \pi^{r}, \quad \text { with } b, c \in V .
$$

Considering the classes of $b$ and $c$ in $S / \mathfrak{M}$, we show that $\bar{b} \neq \bar{c}$ implies $\bar{e}_{r}(x) \neq \bar{e}_{r}(y)$.

1) We first note that, for $\bar{b} \neq 0, \bar{e}_{r}(x) \neq 0$. Indeed, $a_{0}, \ldots, a_{q_{r}-1}, x$ are then in distinct classes modulo $\mathfrak{M}^{r+1}$. They thus form the beginning of a very well distributed sequence, and hence this sequence is a $v$-ordering. Then

$$
v\left(\prod_{0 \leq i<q_{r}}\left(a_{q_{r}}-a_{i}\right)\right)=v\left(\prod_{0 \leq i<q_{r}}\left(x-a_{i}\right)\right) .
$$

Consequently, $v\left(e_{r}(x)\right)=0$, and $\bar{e}_{r}(x) \neq 0$.

If $\bar{c}=0$, as $\bar{e}_{r}$ is constant on cosets modulo $\mathfrak{M}^{r+1}$, we have $\bar{e}_{r}(y)=$ $\bar{e}_{r}\left(a_{j}\right)=0$, and so $\bar{e}_{r}(y) \neq \bar{e}_{r}(x)$. Similarly, if $\bar{b}=0$ and $\bar{c} \neq 0$, we have again $\bar{e}_{r}(y)=0$ and $\bar{e}_{r}(x) \neq 0$.

2) Now we suppose that $\bar{b} \neq 0$ and $\bar{c} \neq 0$. Then $\bar{e}_{r}(x) \neq 0$ and $\bar{e}_{r}(y) \neq 0$. We have

$$
\frac{e_{r}(x)}{e_{r}(y)}=\frac{x-a_{j}}{y-a_{j}} \cdot \prod_{0 \leq k<q_{r}, k \neq j} \frac{x-a_{k}}{y-a_{k}} .
$$

For $k \neq j$,

$$
\frac{x-a_{k}}{y-a_{k}}=1+\frac{x-y}{y-a_{k}}
$$


As $v(x-y)=r$ and $v\left(y-a_{k}\right)<r$, it follows that $\frac{x-y}{y-a_{k}}$ is in $V$ and

$$
\frac{x-a_{k}}{y-a_{k}} \equiv 1(\bmod \mathfrak{M}) \text {. }
$$

On the other hand,

$$
\frac{x-a_{j}}{y-a_{j}}=\frac{b}{c}
$$

As $V$ is local and $c \notin \mathfrak{M}$, it follows that $\frac{b}{c}$ is an element of $V$, thus so is $\frac{e_{r}(x)}{e_{r}(y)}$ and

$$
\frac{e_{r}(x)}{e_{r}(y)} \equiv \frac{b}{c}(\bmod \mathfrak{M}) .
$$

Now, $\bar{b} \neq \bar{c}$ implies $\frac{\bar{b}}{\bar{c}} \neq 1$, hence $\frac{\bar{e}_{r}(x)}{\bar{e}_{r}(y)} \neq 1$, that is, $\bar{e}_{r}(x) \neq \bar{e}_{r}(y)$.

\section{References}

[1] Y. Amice, Interpolation p-adique, Bull. Soc. Math. France 92 (1964), 117-180.

[2] —, Les nombres p-adiques, Presses Univ. de France, Paris, 1975.

[3] M. Bhargava, P-orderings and polynomial functions on arbitrary subsets of Dedekind rings, J. Reine Angew. Math. 490 (1997), 101-127.

[4] M. Bhargava and K. S. Kedlaya, Continuous functions on compact subsets of local fields, Acta Arith. 91 (1999), 191-198.

[5] P. J. Cahen and J. L. Chabert, Integer-Valued Polynomials, Math. Surveys Monogr. 48, Amer. Math. Soc., Providence, 1997.

[6] K. Conrad, The digit principle, J. Number Theory 84 (2000), 230-257.

[7] S. Evrard and Y. Fares, $p$-adic subsets whose factorials satisfy a generalized Legendre formula, Bull. London Math. Soc. 40 (2008), 3750.

[8] A. M. Robert, A Course in p-Adic Analysis, Grad. Texts in Math. 198, Springer, 2000.

[9] J. F Voloch, Differential operators and interpolation series in power series field, J. Number Theory 71 (1998), 106-108.

LAMFA CNRS UMR 6140

Université de Picardie

80039 Amiens, France

E-mail: sabine.evrard@u-picardie.fr

Received on 14.1.2008

and in revised form on 5.9.2008 